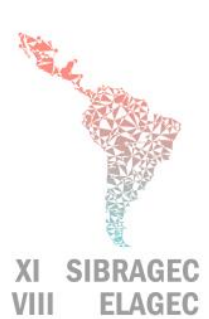

VIII ELAGEC
XI SIMPÓSIO BRASILEIRO DE GESTÃO E ECONOMIA DA CONSTRUÇÃO

VIII ENCUENTRO LATINOAMERICANO DE GESTIÓN Y ECONOMÍA DE LA CONSTRUCCIÓN

Del conocimiento a la acción: prácticas avanzadas de gestión de la producción

Londrina, Paraná, Brasil. 23 al 25 de Octubre del 2019

\title{
REVISIÓN A LA APLICACIÓN DEL LAST PLANNER SYSTEM EN EMPRESAS DE CONSTRUCCIÓN COLOMBIANAS
}

\author{
HOYOS, Maria Fernanda (1); BOTERO, Luis Fernando (2)
}

(1) Universidad EAFIT, e-mail: mhoyosr3@eafit.edu.co

(2) Universidad EAFIT, e-mail: 1fbotero@eafit.edu.co

\begin{abstract}
This article aims to expose the review of the Last Planner System application in three Colombian construction companies. The paper summarizes the second part of the research work of the authors, who in a first stage studied the state of the art of the LPS in Colombia, with the participation of 16 companies. Data collection for the initial diagnosis was made through non-participant observation in the weekly planning meetings of the building projects and dialogue with the managers of the Lean area in each company. The weak aspects in each planning level were all identified, then the socialization of the good practices and permanent feedback was carried out through an accompanying work guided by the authors and the managers of the Lean area. The results show the state of application of good practices and the barriers found by the authors in the interiorization process within the corporate culture.
\end{abstract}

Keywords: Last Planner System, planning, construction management, Colombia.

\section{INTRODUCCIÓN}

El Last Planner System (LPS) se define como un sistema de planificación y control que busca optimizar recursos y disminuir la variabilidad e imprevistos en la industria de la construcción. La metodología nació a principios de los noventa y se consolidó con la tesis doctoral de Ballard (2000). El gremio constructor colombiano, predominantemente tradicionalista, comenzó a aplicarla hace poco más de una década.

El objetivo de los autores fue acompañar a tres empresas constructoras colombianas que aplicaran el LPS como sistema de planificación y control en sus obras, con el fin de identificar sus prácticas, mejorar su apropiación y proponer indicadores en los diferentes niveles de planificación para medir el desempeño de los proyectos.

\section{LAST PLANNER SYSTEM}

El LPS define tres niveles de planificación: general, intermedio y corto plazo. El plan general, define la estrategia de ejecución mediante paquetes de trabajo, teniendo en cuenta fechas de comienzo y fin, relación de dependencia entre ellos y un adecuado estudio de layout. El plan intermedio busca reducir la incertidumbre, determinando con anticipación las restricciones de las actividades, para eliminarlas y garantizar su ejecución oportuna. Finalmente, la planificación semanal es el proceso colaborativo

HOYOS, M.F.; BOTERO, L.F. Revisión a la aplicación del last planner system en empresas de construcción colombianas. In: SIMPÓSIO BRASILEIRO DE GESTÃO E ECONOMIA DA 
donde el equipo de trabajo evalúa el trabajo ejecutado la semana anterior y define las asignaciones para la semana siguiente, identificando las causas de no cumplimiento (CNC) y discutiendo sobre la disponibilidad de recursos en las unidades de actuación.

En la década del noventa se dieron a conocer los primeros casos de estudio donde se expusieron los resultados obtenidos tras la aplicación del LPS en Venezuela (BALLARD et al., 1996), Estados Unidos (BALLARD, 1997; 1999) y Brasil (ITRI, 1998; JUNIOR et al., 1998; MENDES E HEINECK, 1999). Actualmente, el LPS se ha aplicado en numerosos países alrededor del mundo; identificándose barreras de tipo organizacional como la insuficiente preparación de las reuniones de planificación (ALARCÓN et al., 2002); la implementación parcial o intermitente (ALARCÓN et al., 2002; MCCONAUGHY E SHIRKEY, 2013); la falta de integración de la cadena de producción (ALARCÓN et al., 2008; KHANH E KIM, 2015); la falta de vinculación entre los niveles de planificación (HAMZEH et al., 2008; 2012) y el exceso de compromisos sobre el trabajo que puede hacerse desde la planificación intermedia (KHANH E KIM, 2015). De tipo cultural, se resaltan dificultades como la falta de entrenamiento y la autocrítica (ALARCÓN et al., 2002; ALARCÓN et al., 2008); la visión cortoplacista (ALARCÓN et al., 2002; ALSEHAIMI et al., 2009); la resistencia al cambio (ALARCÓN et al., 2002; SHANG E PHENG, 2014; HAMZEH et al., 2016) y el escepticismo frente a los beneficios de la implementación (AHIAKWO et al., 2013).

En Colombia, los autores han identificado la falta de retroalimentación y claridad conceptual, la ausencia del nivel intermedio de planificación y la carencia de comunicación efectiva como las principales falencias en muchas de las compañías que han hecho el esfuerzo de implementar el LPS en su cultura empresarial. Además, la carancia de formalización de procedimientos y buenas prácticas, ha conducido a tener diferentes esquemas y ha dificultado la determinación del impacto real del LPS en el desempeño de los proyectos.

\section{METODOLOGÍA DE TRABAJO}

El trabajo comenzó en 2017 y se extendió por 6 meses en las empresas 1, 2 y 3 (ver Tabla 1). En las primeras visitas los autores conocieron la forma de trabajo de cada empresa y la manera en que abordaban los niveles de planificación; asistieron como observadores no participantes en algunas reuniones de planificación semanal y conocieron, con el apoyo del profesional encargado del área Lean, las herramientas computacionales y procedimientos de cada compañía. Una vez diagnosticado el estado inicial y después de haber identificado los puntos débiles en cada etapa de planificación, se trabajó en el proceso de socialización de buenas prácticas y el mejoramiento de formatos y procedimientos.

\section{Tabla 1 - Resumen de las empresas colaboradoras}

\begin{tabular}{|c|c|c|c|}
\hline Constructora & Antigüedad & Sitio de operación & Mercado \\
\hline 1 & 10 años & Antioquia & Hotelería, oficinas, vivienda \\
\hline 2 & 16 años & $\begin{array}{c}\text { Caldas, Cundinamarca, Huila, } \\
\text { Risaralda, Valle del Cauca }\end{array}$ & $\begin{array}{c}\text { Bodegas, industrial, institucional, } \\
\text { vivienda }\end{array}$ \\
\hline 3 & 1 año & Antioquia & Comercio, hotelería, vivienda \\
\hline
\end{tabular}


SIBRAGEC - ELAGEC 2019 - del 23 al 25 de Octubre - LONDRINA - PR

\section{DIAGNÓSTICO INICIAL}

En la empresa 1 se estudiaron cinco proyectos; en la compañía 2, seis proyectos. En la compañía 3, razón social creada en 2017, se estudió la obra piloto. A partir de la observación no participante y el diálogo con los encargados del área Lean se realizó el diagnóstico inicial de las empresas, que se resume en la Tabla 2.

\section{Tabla 2 - Diagnóstico inicial}

\begin{tabular}{|c|c|}
\hline Aspecto & Diagnóstico \\
\hline Plan general & $\begin{array}{l}\text { Empresa 1: elaborado con Teoría de Restricciones (programación por bloques). } \\
\text { En paralelo se hace el diagrama de Gantt en GanttProject. } \\
\text { Empresa 2: elaborado con líneas de balance en Excel. } \\
\text { Empresa 3: elaborado con Microsoft Project. Se realiza en paralelo líneas de } \\
\text { balance en Excel. }\end{array}$ \\
\hline $\begin{array}{c}\text { Plan } \\
\text { intermedio }\end{array}$ & $\begin{array}{l}\text { Empresa 1: actualizado el plan general se actualiza el plan intermedio } \\
\text { automáticamente. Horizonte de tiempo de seis semanas. Las reuniones de } \\
\text { planificación intermedia no hacen parte de la cultura empresarial. } \\
\text { Empresas } 2 \text { y 3: no existe la cultura de planificación intermedia, claridad sobre } \\
\text { los tiempos de reacción de las áreas de apoyo, ni un formato definido. }\end{array}$ \\
\hline Plan semanal & $\begin{array}{l}\text { Empresas } 1 \text { y 2: reunión semanal rutinaria con subcontratistas. La calificación } \\
\text { del porcentaje del plan completado (PPC) se prepara previo a la reunión. } \\
\text { Escasa discusión de las CNC, los subcontratistas tienen un rol pasivo. } \\
\text { Empresa 1: el registro se hace en formato en Excel estandarizado. } \\
\text { Empresa 2: el registro se hace en Excel sin estandarización. } \\
\text { Empresa 3: no hay un orden del día para la reunión ni formato estandarizado } \\
\text { para el registro de información. }\end{array}$ \\
\hline $\begin{array}{c}\text { Calificación } \\
\text { subcontratistas }\end{array}$ & $\begin{array}{l}\text { Empresa 1: además del PPC, se califican subjetivamente: calidad, gestión } \\
\text { ambiental, manejo de materiales y seguridad. La cartelera de desempeño } \\
\text { muestra la calificación semanal ponderada del subcontratista. } \\
\text { Empresa 2: además del PPC, se califican subjetivamente: calidad, seguridad, } \\
\text { orden y aseo, y recursos. No existe cartelera de desempeño. } \\
\text { Empresa 3: se socializa el PPC verbalmente. No existe cartera de desempeño. }\end{array}$ \\
\hline $\begin{array}{l}\text { Indicadores } \\
\text { existentes }\end{array}$ & $\begin{array}{l}\text { Empresas } 1 \text { y 2: PPC semanal. Sin indicadores del plan general e intermedio. } \\
\text { Empresa 3: hay socialización del PPC, pero sin registro formal. Sin indicadores } \\
\text { del plan general e intermedio. }\end{array}$ \\
\hline $\begin{array}{l}\text { Obstáculos en } \\
\text { la aplicación }\end{array}$ & $\begin{array}{l}\text { Empresa 1: problemas de actualización del software empresarial. El plan } \\
\text { intermedio se realiza a nivel de detalle de asignaciones semanales, generando } \\
\text { un número de actividades difícil de controlar. Diferencias entre el horizonte de } \\
\text { tiempo y los tiempos de reacción de la compañía. } \\
\text { Empresa 2: no hay liberación oportuna de restricciones, las actividades } \\
\text { semanales son programadas de acuerdo con lo ejecutado en obra. } \\
\text { Empresa 3: no hay cultura empresarial y el compromiso de los miembros del } \\
\text { equipo no es constante. Falta de planos completos de la obra, se dificulta la } \\
\text { programación de actividades. }\end{array}$ \\
\hline
\end{tabular}

\section{FASE DE ACOMPAÑAMIENTO}

Se trabajó en el mejoramiento de las herramientas, formatos de registro y calificación integral de subcontratistas a través de la socialización de las buenas prácticas que se resumen en la Ilustración 1. Además, se propuso la implementación de algunos indicadores de desempeño (ver Ilustración 2). Los formatos fueron construidos de forma 
SIBRAGEC - ELAGEC 2019 - del 23 al 25 de Octubre - LONDRINA - PR

mancomunada entre los autores y el equipo Lean, sin embargo, aspectos como las variables de calificación al subcontratista y su ponderación, fueron decisión autónoma de cada empresa.

\section{Ilustración 1 - Buenas prácticas implementadas}

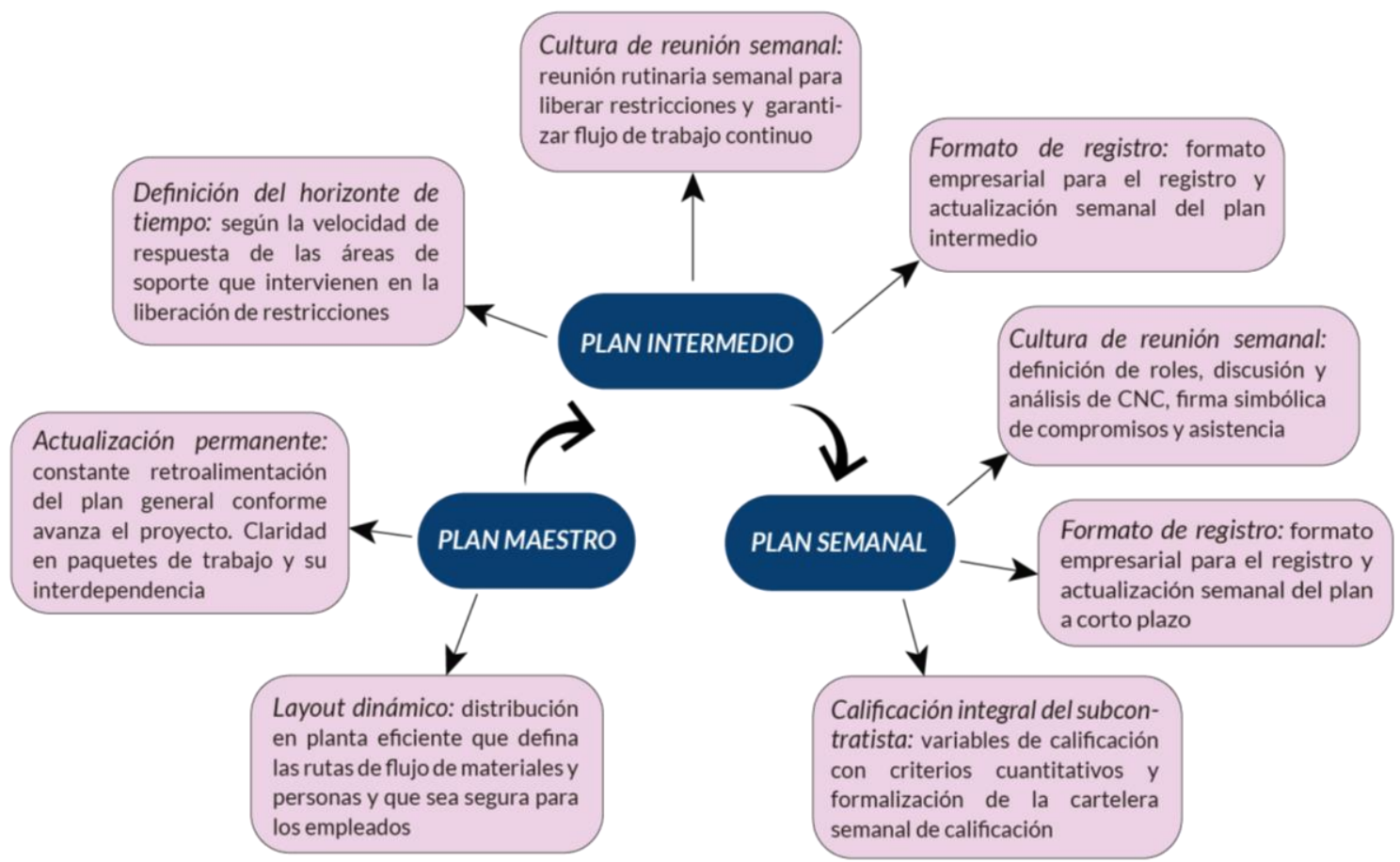

Fuente: elaboración propia

\section{Ilustración 2 - Indicadores propuestos}
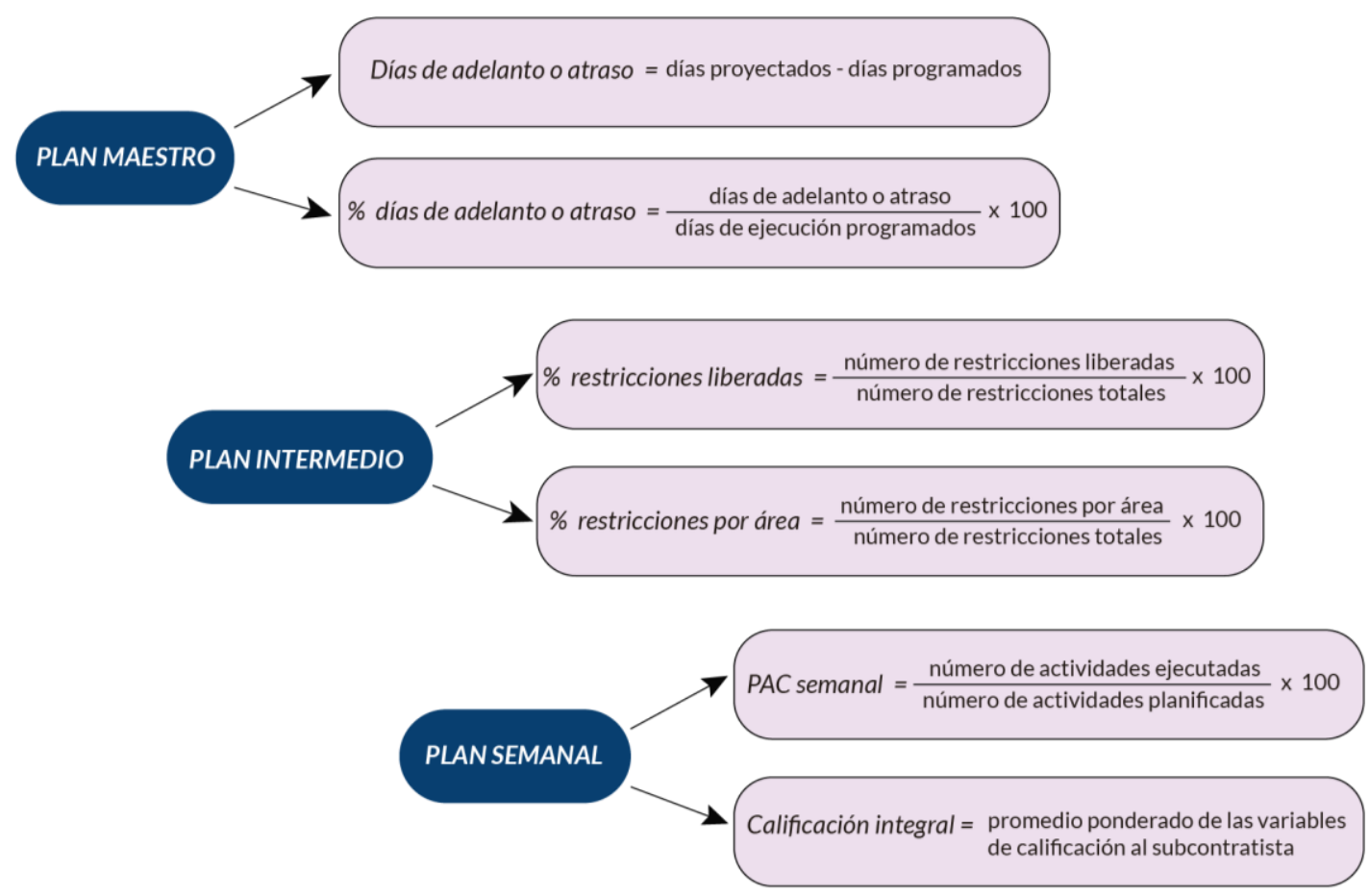

Fuente: elaboración propia 
SIBRAGEC - ELAGEC 2019 - del 23 al 25 de Octubre - LONDRINA - PR

\section{RESULTADOS}

Las buenas prácticas socializadas se impementan actualmente en cada empresa. Los indicadores de desempeño se definieron, sin embargo, al término del trabajo de campo, no todos habían sido puestos en marcha, o se tenían aún resultados muy prematuros para concluir al respecto. En la Tabla 3 se resumen los resultados del proceso, su estado y los obstáculos encontrados en el proceso de incorporación a la cultura empresarial.

Tabla 3 - Resumen de resultados

\begin{tabular}{|c|c|c|}
\hline Aspecto & Estado & Obstáculo \\
\hline $\begin{array}{l}\text { Actualización } \\
\text { permanente del } \\
\text { plan general }\end{array}$ & $\begin{array}{l}\text { Empresas } 1,2 \text { y } 3 \text { : se implementa y } \\
\text { se tienen resultados. }\end{array}$ & $\begin{array}{l}\text { Empresa 1: personal poco familiarizado } \\
\text { con la programación por bloques. } \\
\text { Empresas } 2 \text { y } 3 \text { : tiempo que toma la } \\
\text { actualización del plan, realizado } \\
\text { manualmente con líneas de balance. }\end{array}$ \\
\hline $\begin{array}{l}\text { Formato de } \\
\text { registro de } \\
\text { planificación } \\
\text { intermedia y } \\
\text { definición } \\
\text { horizonte de } \\
\text { tiempo }\end{array}$ & $\begin{array}{l}\text { Empresa 1: socializado con el } \\
\text { coordinador Lean, implementación } \\
\text { pendiente. } \\
\text { Empresa 2: se desarrolla } \\
\text { informalmente. } \\
\text { Empresa 3: se implementa y se } \\
\text { tienen resultados. Formato con } \\
\text { restricciones detalladas por área y } \\
\text { responsable de liberación. }\end{array}$ & $\begin{array}{l}\text { Empresa 1: funcionamiento del } \\
\text { software empresarial y dificultad para } \\
\text { definir el horizonte de tiempo. } \\
\text { Empresa 2: profesionales de obra } \\
\text { reacios a definir fechas de liberación de } \\
\text { restricciones. } \\
\text { Empresa 3: dificultad para establecer un } \\
\text { horizonte de tiempo para los paquetes } \\
\text { de actividades. }\end{array}$ \\
\hline $\begin{array}{l}\text { Cultura de } \\
\text { reunión plan } \\
\text { intermedio }\end{array}$ & $\begin{array}{l}\text { Empresas } 1 \text { y } 2: \text { se desarrolla } \\
\text { informalmente. } \\
\text { Empresa 3: se implementa y se } \\
\text { tienen resultados. }\end{array}$ & $\begin{array}{l}\text { Empresa 1: funcionamiento y } \\
\text { actualización del software empresarial. } \\
\text { Empresas } 2 \text { y } 3: \text { la reunión no se realiza } \\
\text { rutinariamente, se revisan restricciones } \\
\text { de paquetes de actividades sin definir } \\
\text { fechas máximas de liberación. }\end{array}$ \\
\hline $\begin{array}{l}\text { Formato de } \\
\text { registro de } \\
\text { planificación } \\
\text { semanal }\end{array}$ & $\begin{array}{l}\text { Empresa 1: socializado con el } \\
\text { coordinador Lean, implementación } \\
\text { formal pendiente. } \\
\text { Empresas } 2 \text { y 3: se implementa y se } \\
\text { tienen resultados. }\end{array}$ & $\begin{array}{l}\text { Empresa 1: funcionamiento del } \\
\text { software empresarial. } \\
\text { Empresas 1,2 y 3: adaptación inicial de } \\
\text { profesionales Lean y profesionales de } \\
\text { obra en cuanto al funcionamiento del } \\
\text { formato. }\end{array}$ \\
\hline $\begin{array}{l}\text { Cultura de } \\
\text { reunión plan } \\
\text { semanal }\end{array}$ & $\begin{array}{l}\text { Empresas } 1,2 \text { y } 3 \text { : se implementa } \\
\text { formalmente y se tienen resultados. }\end{array}$ & $\begin{array}{l}\text { Empresas } 1,2 \text { y } 3: \text { no se enfatiza en el } \\
\text { análisis y discusión de las CNC. }\end{array}$ \\
\hline $\begin{array}{l}\text { Calificación } \\
\text { integral a } \\
\text { subcontratistas } \\
\text { y cartelera de } \\
\text { desempeño }\end{array}$ & $\begin{array}{l}\text { Empresas } 1,2 \text { y } 3: \text { se implementa } \\
\text { formalmente y se tienen resultados. }\end{array}$ & $\begin{array}{l}\text { Empresas } 1,2 \text { y } 3: \text { dificultad para } \\
\text { calificar objetivamente a los } \\
\text { subcontratistas. } \\
\text { Empresa 1: quejas por el trabajo extra } \\
\text { que representa esta calificación. } \\
\text { Empresa 2: profesionales reacios a } \\
\text { llevar control de las variables de } \\
\text { calificación. }\end{array}$ \\
\hline
\end{tabular}

\section{CONCLUSIONES Y CONSIDERACIONES FINALES}

Los autores destacan como el punto más débil la creación de cultura respecto a la planificación intermedia. Para las empresas colombianas es difícil establecer con 
rigurosidad las fechas máximas de liberación de restricciones, la velocidad de respuesta de las áreas que intervienen en dicha liberación y la rutina de las reuniones, afectando la articulación de los niveles de planificación.

Se advierte también la falta de claridad conceptual en la mayoría de los profesionales de obra, falta de discusión y ahondamiento en la causa raíz de las CNC semanales y falta de estandarización de la calificación al subcontratista. Respecto a las causas externas, se destacan los inconvenientes con los softwares empresariales, los problemas de flujo de caja y la alta rotación del personal, no solo de trabajadores y subcontratistas, sino también los profesionales de obra.

A partir del trabajo realizado fue posible sensibilizar a las compañías respecto a las buenas prácticas del LPS, sin embargo, es de suma importancia que pueda determinarse el impacto en términos de cumplimiento de plazos y su relación directa con el ahorro en términos monetarios. Se deja la invitación abierta a futuras investigaciones para que profundicen sobre este tema.

\section{REFERENCIAS}

AHIAKWO, O. et al. A Case Study of Last Planner System Implementation in Nigeria. In: ANNUAL CONFERENCE OF THE INTERNATIONAL GROUP FOR LEAN CONSTRUCTION, 21., Fortaleza, 2013. Proceedings... Fortaleza: IGLC, 2013

ALARCÓN, L. F.; DIETHELMAND, S.; ROJO, O. Collaborative Implementation of Lean Planning Systems in Chilean Construction Companies. In: ANNUAL CONFERENCE OF THE INTERNATIONAL GROUP FOR LEAN CONSTRUCTION, 10., Gramado, 2002. Proceedings... Gramado: IGLC, 2002.

ALARCÓN, L. F. et al. Assessing the impacts of implementing lean construction. Revista Ingeniería de Construcción, v. 23, p. 26-33, 2008.

ALSEHAIMI, A.; TZORTZOPOULOS, P.; KOSKELA, L. Last Planner System- Experiences From Pilot Implementation in the Middle East. In: ANNUAL CONFERENCE OF THE INTERNATIONAL GROUP FOR LEAN CONSTRUCTION, 7., Taipei, 2009. Proceedings... Taipei: IGLC, 2009.

BALLARD, G. Lookahead Planning. The Missing Link in Production Control. In: ANNUAL CONFERENCE OF THE INTERNATIONAL GROUP FOR LEAN CONSTRUCTION, 5., Gold Coast, 1997. Proceedings... Gold Coast: IGLC, 1997

Improving work flow reliability. In: ANNUAL CONFERENCE OF THE

INTERNATIONAL GROUP FOR LEAN CONSTRUCTION, 7., Berkeley, 1999. Proceedings... Berkeley: IGLC, 1999.

. The Last Planner System of Production Control. 2000. (Doctor of Philosophy ). School of Civil Engineering, Faculty of Engineering, University of Birmingham, Salford, U.K.

BALLARD, G.; CASTEN, M.; HOWELL, G. PARC: A Case Study. In: ANNUAL CONFERENCE OF THE INTERNATIONAL GROUP FOR LEAN CONSTRUCTION, 4. , Birmingham, 1996. Proceedings... Birmingham: IGLC, 1996.

HAMZEH, F.; BALLARD, G.; TOMMELEIN, I. Improving Construction Work Flow - The Connective Role of Lookahead Planning. In: ANNUAL CONFERENCE OF THE INTERNATIONAL GROUP FOR LEAN CONSTRUCTION, 16., Manchester, 2008. Proceedings... Manchester: IGLC, 2008.

Rethinking Lookahead Planning to Optmize Construction Worflow. Lean

Construction Journal, p. 15-34, 2012. 
SIBRAGEC - ELAGEC 2019 - del 23 al 25 de Octubre - LONDRINA - PR

HAMZEH, F. et al. The First Extensive Implementation of Lean and LPS in Lebanon: Results and Reflections. In: ANNUAL CONFERENCE OF THE INTERNATIONAL GROUP FOR LEAN CONSTRUCTION, 24., Boston, 2016. Proceedings... Boston: IGLC, 2016.

ITRI, A. S. Last Planner, Look Ahead, PPC: a Driver to the Site Operations. In: ANNUAL CONFERENCE OF THE INTERNATIONAL GROUP FOR LEAN CONSTRUCTION, 6. Guarujá, 1998. Proceedings... Guarujá: IGLC, 1998.

JUNIOR, J. A.; SCOLA, A.; ITRI, S. A. Last Planner as a Site Operations Tool. In: ANNUAL CONFERENCE OF THE INTERNATIONAL GROUP FOR LEAN CONSTRUCTION, 6. Guarujá, 1998. Proceedings... Guarujá: IGLC, 1998.

KHANH, H.; KIM, S. A Survey on Production Planning System in Construction Projects Based on Last Planner System. KSCE Journal of Civil Engineering, v. 20, n. 1, p. 1-11, 2015.

MCCONAUGHY, T.; SHIRKEY, D. Subcontractor Collaboration and Breakdowns in Production- The Effects of Varied Lps Implementation. In: ANNUAL CONFERENCE OF THE INTERNATIONAL GROUP FOR LEAN CONSTRUCTION, 21., Fortaleza, 2013.

Proceedings... Fortaleza: IGLC, 2013.

MENDES, R. J.; HEINECK, L. F. M. Towards Production Control on Multi-Story Building Construction Sites. In: ANNUAL CONFERENCE OF THE INTERNATIONAL GROUP FOR LEAN CONSTRUCTION, 7., Berkeley, 1999. Proceedings... Berkeley: IGLC, 1999.

SHANG, G.; PHENG, L. The Last Planner System in China's construction industry - A SWOT analysis on implementation. International Journal of Project Management, v. 32, p. 12601272, 2014. 